\title{
Homeomorphic Changes of Variable and Fourier Multipliers
}

\author{
Vladimir LeBedev And Alexander OlevskiI
}

\begin{abstract}
We consider the algebras $M_{p}$ of Fourier multipliers and show that for every bounded continuous function $f$ on $\mathbb{R}^{d}$ there exists a self-homeomorphism $h$ of $\mathbb{R}^{d}$ such that the superposition $f \circ h$ is in $M_{p}\left(\mathbb{R}^{d}\right)$ for all $p, 1<p<\infty$. Moreover, under certain assumptions on a family $K$ of continuous functions, one $h$ will suffice for all $f \in K$. A similar result holds for functions on the torus $\mathbb{T}^{d}$. This may be contrasted with the known solution of Luzin's problem related to the Wiener algebra.
\end{abstract}

Key words: Fourier multipliers, superposition operators.

2010 Mathematics Subject Classification: Primary 42A45, 42B15.

\section{Introduction}

To what extent the behavior of the Fourier series of a continuous function can be improved by a change of variable? The problem originates from a theorem of Bohr and Pál (see [1, Ch. 4, Sec. 12], [9], [23]), who showed that given a continuous real-valued function $f$ on the circle $\mathbb{T}=\mathbb{R} / 2 \pi \mathbb{Z}$, there exists a self-homeomorphism $h$ of $\mathbb{T}$ such that the Fourier series of the superposition $f \circ h$ converges uniformly. In addition, the proof yields a condition on the decay of the Fourier coefficients of $f \circ h$; namely,

$$
\widehat{f \circ h} \in l^{p}(\mathbb{Z})
$$

for all $p>1$.

This result inspired the following problem posed by N. Lusin (see [1, Ch. 4, Sec. 12], [8, Ch. VII, Sec. 9]): Is it possible to attain condition (1) for $p=1$ ? In other words, is it true that for every continuous function on $\mathbb{T}$ there exists a change of variable which brings it into the Wiener algebra $A(\mathbb{T})$ ?

It is worth noting that the original proof of the Bohr-Pál theorem involves Riemann's theorem on conformal mappings. A purely real-analytic proof was found in [25]. 
A solution (in the negative) of Lusin's problem was obtained in [22], where it is shown that there exists a continuous real-valued function $f$ on $\mathbb{T}$ such that $f \circ h \notin A(\mathbb{T})$ whenever $h$ is a self-homeomorphism of $\mathbb{T}$. Some ideas of P. Cohen were used in the proof. Simultaneously, the same result for a complex-valued $f$ was obtained in [10] (this weaker result amounts to the fact that, in general, there is no single change of variable which will bring two continuous real-valued functions into $A(\mathbb{T}))$.

Subsequently, for certain function spaces, naturally arising in harmonic analysis, the question of whether every continuous function can be transformed by a suitable homeomorphic change of variable into a function that belongs to a given space, was studied by various authors. Some of these studies concern the possibility of simultaneous improvement of several functions by means of a single change of variable; we note in particular the work [11] where the Bohr-Pál theorem is extended to compact families of continuous functions.

For a survey on the subject see [23], [9]. For more recent results see [24], [4, Ch. 9], [15], [16], [18], [19]. We also mention the papers [14], [13] which study the growth of the partial sums of the Fourier series of $f \circ h$ for random homeomorphisms $h$.

In the present paper we investigate the problem on changes of variable in relation with the Fourier Multiplier Algebras. We consider multipliers on $\mathbb{R}^{d}$ and on the torus $\mathbb{T}^{d}$.

Let $G$ be one of the groups $\mathbb{R}^{d}$ or $\mathbb{Z}^{d}$ and let $\Gamma$ be its dual group, i.e., $\Gamma=\mathbb{R}^{d}$ or $\Gamma=\mathbb{T}^{d}$, correspondingly. Consider a function $m \in L^{\infty}(\Gamma)$ and the operator $Q$ defined by

$$
\widehat{Q f}=m \cdot \widehat{f}, \quad f \in L^{p} \cap L^{2}(G),
$$

where ${ }^{\wedge}$ stands for the Fourier transform on $G$. The function $m$ is called an $L^{p}$ multiplier $(1 \leq p \leq \infty)$ if

$$
\|Q f\|_{L^{p}(G)} \leq c\|f\|_{L^{p}(G)}, \quad f \in L^{p} \cap L^{2}(G),
$$

where $c>0$ does not depend on $f$. The space of all such multipliers is denoted by $M_{p}(\Gamma)$. The norm on $M_{p}(\Gamma)$ is defined by setting $\|m\|_{M_{p}(\Gamma)}$ to be the smallest $c$ for which $(3)$ holds. The space $M_{p}(\Gamma)$ equipped with this norm is a Banach algebra with the usual multiplication of functions. Clearly, if $p<\infty$, then the operator $Q$ that corresponds to the function $m$ can be uniquely extended to a bounded operator on $L^{p}(G)$, and retaining 
the notation $Q$ for this extension, we have $\|Q\|_{L^{p}(G) \rightarrow L^{p}(G)}=\|m\|_{M_{p}(\Gamma)}$. We also note that the operator $Q$ is translation-invariant. The converse also holds for $p<\infty$ : every translation-invariant bounded operator on $L^{p}(G)$ has the form $(2)$, where $m \in M_{p}(\Gamma)$. For basic properties of multipliers see $[3],[5]$.

It is known that $M_{2}$ coincides with $L^{\infty}$. It is also known that $M_{1}=M_{\infty}$, and, at the same time, $M_{1}\left(\mathbb{T}^{d}\right)$ coincides with the Wiener algebra $A\left(\mathbb{T}^{d}\right)$ and $M_{1}\left(\mathbb{R}^{d}\right)$ coincides with the algebra $B\left(\mathbb{R}^{d}\right)$ of the Fourier transforms of (complex) bounded regular Borel measures on $\mathbb{R}^{d}$. Note that the negative solution of Luzin's problem immediately implies a similar result for functions on the real line: there exists a bounded continuous real-valued function $f$ on $\mathbb{R}$ such that $f \circ h \notin B(\mathbb{R})$ for every self-homeomorphism $h$ of $\mathbb{R}$ (for details, see Sec. 5). Thus, in general, there is no change of variable which will bring a continuous real-valued function on $\mathbb{T}$ (a bounded continuous function on $\mathbb{R})$ into $M_{1}=M_{\infty}$.

In this paper we show that for every bounded continuous function $f$ on $\mathbb{R}^{d}$ (for every continuous function $f$ on $\mathbb{T}^{d}$ ) there is a homeomorphic change of variable $h$ such that $f \circ h \in \bigcap_{1<p<\infty} M_{p}$. Moreover, under certain natural assumptions on a function family $K$, one change of variable will suffice for all $f \in K$. An important role in the proof is played by a result of Sjögren and Sjölin on Littlewood-Paley partitions.

The exact statements of our results are given in Section 2 below. Section 3 contains preliminaries. The proofs are given in Section 4. The concluding Section 5 contains several remarks and open problems; in particular, we focus on the famous Beurling-Helson theorem and briefly discuss some recent results and open questions related to this theorem and to its version for Multiplier Algebras.

\section{Statement of Results}

Let $f$ be a function defined on a set $E$ (we assume that $E \subseteq \mathbb{R}^{d}$ or $\left.E \subseteq \mathbb{T}^{d}\right)$. By $\omega(f, E, \delta)$ we denote the modulus of continuity of $f$ on $E$ :

$$
\omega(f, E, \delta)=\sup _{\substack{t_{1}, t_{2} \in E \\\left|t_{1}-t_{2}\right| \leq \delta}}\left|f\left(t_{1}\right)-f\left(t_{2}\right)\right|, \quad \delta \geq 0
$$

$\left(|x|\right.$ stands for the length of a vector $\left.x \in \mathbb{R}^{d}\right)$. Let $K$ be a family of functions 
on $E$. We define the modulus of continuity $\omega(K, E, \delta)$ of $K$ on $E$ by

$$
\omega(K, E, \delta)=\sup _{f \in K} \omega(f, E, \delta), \quad \delta \geq 0
$$

We say that $K$ is uniformly equicontinuous on $E$ if $\omega(K, E, \delta) \rightarrow 0$ as $\delta \rightarrow+0$.

By $C\left(\mathbb{R}^{d}\right)$ and $C\left(\mathbb{T}^{d}\right)$ we denote the classes of continuous functions on $\mathbb{R}^{d}$ and $\mathbb{T}^{d}$, respectively. The results of this paper are the following two theorems.

THEOREM 1. Let $f \in C\left(\mathbb{R}^{d}\right)$ be a bounded function. Then there exists a self-homeomorphism $h$ of $\mathbb{R}^{d}$ such that $f \circ h \in \bigcap_{1<p<\infty} M_{p}\left(\mathbb{R}^{d}\right)$. Moreover, if a family $K \subseteq C\left(\mathbb{R}^{d}\right)$ of bounded functions is uniformly equicontinuous on every ball in $\mathbb{R}^{d}$, then there exists a self-homeomorphism $h$ of $\mathbb{R}^{d}$ such that $f \circ h \in \bigcap_{1<p<\infty} M_{p}\left(\mathbb{R}^{d}\right)$ for all $f \in K$.

THEOREM 2. Let $f \in C\left(\mathbb{T}^{d}\right)$. Then there exists a self-homeomorphism $h$ of $\mathbb{T}^{d}$ such that $f \circ h \in \bigcap_{1<p<\infty} M_{p}\left(\mathbb{T}^{d}\right)$. Moreover, if a family $K \subseteq C\left(\mathbb{T}^{d}\right)$ is uniformly equicontinuous on $\mathbb{T}^{d}$, then there exists a self-homeomorphism $h$ of $\mathbb{T}^{d}$ such that $f \circ h \in \bigcap_{1<p<\infty} M_{p}\left(\mathbb{T}^{d}\right)$ for all $f \in K$.

\section{Preliminaries}

In this section we recall some notions and facts from the LittlewoodPaley and multiplier theory.

A. For $1 / p+1 / q=1$ we have $M_{p}(\Gamma)=M_{q}(\Gamma)$ and $\|\cdot\|_{M_{p}}=\|\cdot\|_{M_{q}}$. If $1 \leq p_{1}<p_{2} \leq 2$, then $M_{p_{1}}(\Gamma) \subset M_{p_{2}}(\Gamma)$ and $\|\cdot\|_{M_{p_{2}}} \leq\|\cdot\|_{M_{p_{1}}}$ (see, e.g., $[3])$.

B. The indicator function $1_{I}$ of any rectangle $I \subseteq \mathbb{R}^{d}$ belongs to $M_{p}\left(\mathbb{R}^{d}\right)$ for all $p, 1<p<\infty$, see, e.g., [3]. (As usual, we set $1_{I}(t)=1$ if $t \in I$ and $1_{I}(t)=0$ if $t \notin I$.)

C. Let $m \in M_{p}\left(\mathbb{R}^{d}\right), 1 \leq p \leq \infty$, and let $l: \mathbb{R}^{d} \rightarrow \mathbb{R}^{d}$ be a nondegenerate affine mapping; then $m \circ l \in M_{p}\left(\mathbb{R}^{d}\right)$ and $\|m \circ l\|_{M_{p}\left(\mathbb{R}^{d}\right)}=\|m\|_{M_{p}\left(\mathbb{R}^{d}\right)}$ (see, e.g., [5, Ch. I, Sec. 1.3]). 
D. Let $m_{0}$ be a function in $M_{p}\left(\mathbb{R}^{d}\right), 1 \leq p \leq \infty$, vanishing outside a cube $I$ with edges of length $2 \pi$ parallel to the coordinate axes. Consider the extension $m$ of $m_{0}$ to $\mathbb{R}^{d}$ which is $2 \pi$-periodic in each variable. The function $m$ belongs to $M_{p}\left(\mathbb{T}^{d}\right)$, and $\|m\|_{M_{p}\left(\mathbb{T}^{d}\right)} \leq c_{p}\left\|m_{0}\right\|_{M_{p}\left(\mathbb{R}^{d}\right)}$, where $c_{p}$ is a positive constant not depending on $m_{0}$. This theorem on extension of multipliers is well known [6, Theorem 2.3] (for $p=1, \infty$ the result follows from the local properties of the Fourier transforms of measures, see., e.g., [8, Ch. II, Sec. 4]).

E. Let $I \subseteq \mathbb{R}^{d}$ be a rectangle. By $S_{I}$ we denote the operator on $L^{p}\left(\mathbb{R}^{d}\right)$ that corresponds to multiplication by $1_{I}$, i.e., the operator defined by

$$
\widehat{S_{I}(f)}=1_{I} \cdot \widehat{f}, \quad f \in L^{p} \cap L^{2}\left(\mathbb{R}^{d}\right) .
$$

Let $\Delta$ be a family of rectangles which form a partition of $\mathbb{R}^{d}$, i.e., a family of pairwise disjoint rectangles in $\mathbb{R}^{d}$ such that the complement $\mathbb{R}^{d} \backslash \bigcup_{I \in \Delta} I$ has Lebesgue measure zero. Consider the corresponding Littlewood-Paley square function $S^{\Delta}(f)$ :

$$
S^{\Delta}(f)=\left(\sum_{I \in \Delta}\left|S_{I}(f)\right|^{2}\right)^{1 / 2} .
$$

The partition $\Delta$ is called an LP partition if, for all $p, 1<p<\infty$, we have

$$
a_{p} \cdot\|f\|_{L^{p}\left(R^{d}\right)} \leq\left\|S^{\Delta}(f)\right\|_{L^{p}\left(R^{d}\right)} \leq b_{p} \cdot\|f\|_{L^{p}\left(R^{d}\right)},
$$

where $a_{p}=a_{p}(\Delta)$ and $b_{p}=b_{p}(\Delta)$ are positive constants independent of $f$. A classical example of an LP partition of the real line $\mathbb{R}$ is the dyadic partition, that is the family of the intervals $I_{k}, k \in \mathbb{Z}$, of the form $I_{k}=\left(2^{k-1}, 2^{k}\right)$ for $k=1,2, \ldots, I_{0}=(-1,1)$, and $I_{k}=\left(-2^{-k},-2^{-k-1}\right)$ for $k=-1,-2, \ldots$

Let $\Delta$ be a family of rectangles which form an LP partition of $\mathbb{R}^{d}$. Consider an arbitrary function $m \in L^{\infty}\left(\mathbb{R}^{d}\right)$ constant on each rectangle $I \in \Delta$. Then $m$ is in $M_{p}\left(\mathbb{R}^{d}\right)$ for all $p, 1<p<\infty$, and

$$
\|m\|_{M_{p}\left(\mathbb{R}^{d}\right)} \leq c(p, \Delta) \cdot\|m\|_{L^{\infty}\left(\mathbb{R}^{d}\right)},
$$

where $c(p, \Delta)>0$ does not depend on $m$ (see, e.g., $[3,1.2 .7,1.2 .8]$ ).

Given a family $\Delta$ of intervals in $\mathbb{R}$, let $\Delta_{d}$ denote the family of rectangles in $\mathbb{R}^{d}$ generated by $\Delta$, that is, the family of all rectangles $I$ of the form 
$I=I_{1} \times I_{2} \times \ldots I_{d}$, where each factor belongs to $\Delta$. If $\Delta$ is an LP partition of $\mathbb{R}$, then $\Delta_{d}$ is an LP partition of $\mathbb{R}^{d}$ (see [3, Theorem 1.3.4]).

F. By the dyadic partition of the interval $(0,1)$ we mean the family of the intervals $I_{k}, k \in \mathbb{Z}$, defined by

$$
\begin{gathered}
I_{k}=\left(1-2^{-k-1}, 1-2^{-k-2}\right), \quad k=1,2, \ldots ; \\
I_{0}=(1 / 4,3 / 4) ; \\
I_{k}=\left(2^{k-2}, 2^{k-1}\right), \quad k=-1,-2, \ldots .
\end{gathered}
$$

We extend this definition to any interval $(a, b)$ in the obvious way by translation and rescaling. Suppose now that $\Delta$ is a family of intervals which form an LP partition of $\mathbb{R}$. Then the family of all intervals, obtained by dyadic partition of each interval $I \in \Delta$ is an LP partition of $\mathbb{R}$ as well. This immediately follows from a result of Sjögren and Sjölin [26, Theorem 1.2].

\section{Proofs of the Theorems}

First, we prove a simple auxiliary lemma.

Lemma. Let $f \in C\left(\mathbb{R}^{d}\right)$. Then there exists a self-homeomorphism $\psi$ of $\mathbb{R}^{d}$ such that $f \circ \psi$ is uniformly continuous on $\mathbb{R}^{d}$. Moreover, if a family $K \subseteq C\left(\mathbb{R}^{d}\right)$ is uniformly equicontinuous on every ball in $\mathbb{R}^{d}$, then there exists a self-homeomorphism $\psi$ of $\mathbb{R}^{d}$ such that the family $\{f \circ \psi, f \in K\}$ is uniformly equicontinuous on $\mathbb{R}^{d}$.

Proof. We give the proof in the general case of families of functions. Consider spherical shells

$$
\Theta_{j}=\left\{x \in \mathbb{R}^{d}: j \leq|x| \leq j+1\right\}, \quad j=0,1,2, \ldots
$$

$\left(\Theta_{0}\right.$ is a ball). Let $\omega_{j}$ be the modulus of continuity of the family $K$ on $\Theta_{j}$, i.e.,

$$
\omega_{j}(\delta)=\sup _{f \in K} \omega\left(f, \Theta_{j}, \delta\right) .
$$

By assumption, $\omega_{j}(\delta) \rightarrow 0$ as $\delta \rightarrow+0$ for each $j$. So, we can find a decreasing sequence $b_{j}, j=0,1,2, \ldots$, such that $0<b_{j}<1$ and

$$
\omega_{j}\left(b_{j}\right) \rightarrow 0 \text { as } \quad j \rightarrow \infty
$$


We set

$$
a_{j}=\frac{b_{j}}{j+2}, \quad j=1,2, \ldots
$$

The sequence $a_{j}, j=1,2, \ldots$, decreases, and $0<a_{j}<1$. Define numbers $r_{j}, j=0,1,2, \ldots$, by

$$
r_{0}=0 ; \quad r_{j}=\sum_{s=1}^{j} \frac{1}{a_{s}}, \quad j=1,2, \ldots
$$

Clearly, there exists a function $g$ on $[0,+\infty)$ with the following properties:

(i) $g(0)=0$;

(ii) $g$ is continuous and strictly increasing;

(iii) for each $j=0,1,2, \ldots$ the function $g$ is linear on the interval $\left[r_{j}, r_{j+1}\right]$ and maps $\left[r_{j}, r_{j+1}\right]$ onto the interval $[j, j+1]$;

(iv) the slope of $g$ on $\left[r_{j}, r_{j+1}\right]$ equals $a_{j+1}$ (see (5)).

For $x \in \mathbb{R}^{d}$, we set

$$
\psi(x)=g(|x|) \frac{x}{|x|}, \quad x \neq 0 ; \quad \psi(0)=0 .
$$

One can easily see that $\psi$ is a self-homeomorphism of $\mathbb{R}^{d}$.

Consider the spherical shells

$$
\Omega_{j}=\left\{x: r_{j} \leq|x| \leq r_{j+1}\right\}, \quad j=0,1,2, \ldots
$$

$\left(\Omega_{0}\right.$ is a ball). Note, that the image of $\Omega_{j}$ under $\psi$ is the shell $\Theta_{j}$. We claim that

$$
|\psi(x)-\psi(y)| \leq b_{j}|x-y| \quad \text { for all } \quad x, y \in \Omega_{j}, \quad j=0,1,2, \ldots
$$

Since $\psi(x)=a_{1} x$ on $\Omega_{0}$, we see that if $x, y \in \Omega_{0}$, then

$$
|\psi(x)-\psi(y)|=a_{1}|x-y|=\left(b_{1} / 3\right)|x-y| \leq b_{0}|x-y| .
$$

Let $j \geq 1$. Observe that the mapping $\gamma(x)=x /|x|$ has the property that $\left|\gamma\left(x_{1}\right)-\gamma\left(x_{2}\right)\right| \leq(1 / r)\left|x_{1}-x_{2}\right|$ for all $x_{1}, x_{2}$ that lie outside the ball $B_{r}=$ $\{x:|x|<r\}, r>0$. So, if $x, y \in \Omega_{j}$, then

$$
|\gamma(x)-\gamma(y)| \leq \frac{1}{r_{j}}|x-y| .
$$


At the same time

$$
|g(|x|)-g(|y|)|=a_{j+1}|| x|-| y|| \leq a_{j+1}|x-y| .
$$

Whence, taking into account that $g(|y|) \leq j+1$ and $r_{j} \geq 1 / a_{j}$, we obtain

$$
\begin{aligned}
& |\psi(x)-\psi(y)|=|(g(|x|)-g(|y|)) \gamma(x)+g(|y|)(\gamma(x)-\gamma(y))| \\
\leq & a_{j+1}|x-y|+(j+1) \frac{1}{r_{j}}|x-y| \leq(j+2) a_{j}|x-y|=b_{j}|x-y| .
\end{aligned}
$$

Thus, (6) holds.

Let $f \in K$. We shall estimate the modulus of continuity of the superposition $f \circ \psi$ on $\mathbb{R}^{d}$. Let $0<\delta \leq 1$, and let $x, y \in \mathbb{R}^{d},|x-y| \leq \delta$. Since the thickness of each shell $\Omega_{j}$ is greater than 1 (it equals $1 / a_{j+1}$ ), we see that either the points $x$ and $y$ belong to the same shell, say $\Omega_{j}$, or there are two neighboring shells $\Omega_{j}$ and $\Omega_{j+1}$ such that $x$ is in one of them and $y$ is in the other one. Consider the first case when $x$ and $y$ are in $\Omega_{j}$. Then the points $\psi(x), \psi(y)$ belong to the shell $\Theta_{j}$. So (see (6)),

$$
|f \circ \psi(x)-f \circ \psi(y)| \leq \omega_{j}(|\psi(x)-\psi(y)|) \leq \omega_{j}\left(b_{j} \delta\right) .
$$

Consider the second case when $x \in \Omega_{j}$ and $y \in \Omega_{j+1}$. The line segment that joins $x$ and $y$ contains a point $z$ that belongs to both $\Omega_{j}$ and $\Omega_{j+1}$. Using the estimate obtained in the first case, we have

$|f \circ \psi(x)-f \circ \psi(y)| \leq|f \circ \psi(x)-f \circ \psi(z)|+|f \circ \psi(z)-f \circ \psi(y)| \leq \omega_{j}\left(b_{j} \delta\right)+\omega_{j+1}\left(b_{j+1} \delta\right)$.

Thus, we see that for $0<\delta \leq 1$

$$
\omega\left(f \circ \psi, \mathbb{R}^{d}, \delta\right) \leq 2 \sup _{j \geq 0} \omega_{j}\left(b_{j} \delta\right) .
$$

To complete the proof it remains to note that (4) implies

$$
\sup _{j \geq 0} \omega_{j}\left(b_{j} \delta\right) \rightarrow 0 \quad \text { as } \quad \delta \rightarrow+0 .
$$

Proof of Theorem 1. Let $I=(l, r)$ be a bounded or unbounded interval in $\mathbb{R}$. We say that intervals $I_{k} \subset I, k \in \mathbb{Z}$, form an ordered partition of $I$ if $I_{k}=\left(\theta_{k}, \theta_{k+1}\right)$, where $\theta_{k}<\theta_{k+1}$ for all $k \in \mathbb{Z}$, $\lim _{k \rightarrow+\infty} \theta_{k}=r$, and $\lim _{k \rightarrow-\infty} \theta_{k}=l$. 
Suppose that intervals $I_{s_{1}}, s_{1} \in \mathbb{Z}$, form an ordered partition of $\mathbb{R}$. For each fixed $s_{1}$, let $I_{s_{1}, s_{2}}, s_{2} \in \mathbb{Z}$, be certain intervals, which form an ordered partition of $I_{s_{1}}$, and for each $\nu$ and integers $s_{1}, s_{2}, \ldots, s_{\nu}$ let $I_{s_{1}, s_{2}, \ldots, s_{\nu}, s_{\nu+1}}$, $s_{\nu+1} \in \mathbb{Z}$, be intervals, which form an ordered partition of $I_{s_{1}, s_{2}, \ldots, s_{\nu}}$. Proceeding, we obtain a certain family of intervals:

$$
\left\{I_{s_{1}, s_{2}, \ldots, s_{\nu}}: \nu=1,2, \ldots, \quad \text { and } \quad s_{1}, s_{2}, \ldots, s_{\nu} \in \mathbb{Z}\right\}
$$

We refer to any family of intervals thus obtained as a net. For each fixed $\nu$, the intervals $I_{s_{1}, s_{2}, \ldots, s_{\nu}}$ are called intervals of rank $\nu$ (of a given net).

Given a set $E$ and a function $f$ on $E$, by $\operatorname{osc}_{E} f$ we denote the oscillation of $f$ on $E$ : $\operatorname{osc}_{E} f=\sup _{t_{1}, t_{2} \in E}\left|f\left(t_{1}\right)-f\left(t_{2}\right)\right|$.

We shall construct two nets of intervals. Clearly, the dyadic partition of the real line or of an interval (see Section 3, E and F) is an ordered partition. Consider the intervals $I_{s_{1}}, s_{1} \in \mathbb{Z}$, which form the dyadic partition of $\mathbb{R}$. If intervals $I_{s_{1}, s_{2}, \ldots, s_{\nu}}$ of rank $\nu$ are already defined, then we define $I_{s_{1}, s_{2}, \ldots, s_{\nu}, s_{\nu+1}}$, $s_{\nu+1} \in \mathbb{Z}$, to be the intervals that form the dyadic partition of $I_{s_{1}, s_{2}, \ldots, s_{\nu}}$. Thereby, we have constructed the first net, which we denote by $\alpha$.

By $\alpha_{d}(\nu)$ we denote the family of all rectangles in $\mathbb{R}^{d}$ obtained as the Cartesian product of any $d$ intervals of rank $\nu$ of the net $\alpha$. It follows from the properties of LP partitions listed in Section 3, E and F, that if $m$ is a function in $L^{\infty}\left(\mathbb{R}^{d}\right)$ constant on each rectangle which belongs to $\alpha_{d}(\nu)$, then $m \in M_{p}\left(\mathbb{R}^{d}\right)$ for all $p, 1<p<\infty$, and

$$
\|m\|_{M_{p}\left(\mathbb{R}^{d}\right)} \leq c(p, \nu) \cdot\|m\|_{L^{\infty}\left(\mathbb{R}^{d}\right)}, \quad 1<p<\infty
$$

where $c(p, \nu)>0$ may depend only on $p$ and $\nu$. In what follows, we assume that

$$
c(p, \nu)=\sup _{m \in \mathcal{P}_{\nu}, m \neq 0} \frac{\|m\|_{M_{p}\left(\mathbb{R}^{d}\right)}}{\|m\|_{L^{\infty}\left(\mathbb{R}^{d}\right)}},
$$

where $\mathcal{P}_{\nu}$ is the class of all functions in $L^{\infty}\left(\mathbb{R}^{d}\right)$ constant on each rectangle from $\alpha_{d}(\nu)$.

We proceed to the construction of the second net. By using the Lemma, we can assume that $K$ is a family of bounded functions uniformly equicontinuous on the whole $\mathbb{R}^{d}$. Let $\omega$ be the modulus of continuity of $K$. We have

$$
\sup _{\substack{t_{1}, t_{2} \in \mathbb{R}^{d} \\\left|t_{1}-t_{2}\right| \leq \delta}}\left|f\left(t_{1}\right)-f\left(t_{2}\right)\right| \leq \omega(\delta), \quad \delta>0, \quad \text { for all } f \in K
$$


where $\omega(\delta) \rightarrow 0$ as $\delta \rightarrow+0$, and $\omega$ is nondecreasing on $[0,+\infty)$. Fix a positive decreasing sequence $\delta_{\nu}, \nu=1,2, \ldots$, that tends to 0 so fast that

$$
\sum_{\nu=2}^{\infty} c\left(1+\frac{1}{\nu}, \nu\right) \omega\left(\delta_{\nu-1} \sqrt{d}\right)<\infty .
$$

Note that (8) implies that

$$
\sum_{\nu=2}^{\infty} c(p, \nu) \omega\left(\delta_{\nu-1} \sqrt{d}\right)<\infty
$$

for all $p, 1<p<\infty$. Indeed, it suffices to observe that if $\nu$ is large enough, then $c(p, \nu) \leq c(1+1 / \nu, \nu)$ (see Section $3, \mathrm{~A})$.

Let $J_{s_{1}}, s_{1} \in \mathbb{Z}$, be intervals of length at most $\delta_{1}$ which form an ordered partition of the line $\mathbb{R}$. For a fixed $\nu$, assuming that all the intervals $J_{s_{1}, s_{2}, \ldots, s_{\nu}}$ are already defined, consider an ordered partition of each interval $J_{s_{1}, s_{2}, \ldots, s_{\nu}}$ by intervals $J_{s_{1}, s_{2}, \ldots, s_{\nu}, s_{\nu+1}}, s_{\nu+1} \in \mathbb{Z}$, of length at most $\delta_{\nu+1}$. Thereby, we obtain the second net, which we denote by $\beta$.

Clearly, there exists a self-homeomorphism $\varphi$ of $\mathbb{R}$ such that $\varphi\left(I_{s_{1}, s_{2}, \ldots, s_{\nu}}\right)=$ $J_{s_{1}, s_{2}, \ldots, s_{\nu}}$ for all $\nu=1,2, \ldots$ and $s_{1}, s_{2}, \ldots, s_{\nu}$ (the intervals $I_{s_{1}, s_{2}, \ldots, s_{\nu}}$ and $J_{s_{1}, s_{2}, \ldots, s_{\nu}}$ belong to the nets $\alpha$ and $\beta$, respectively). We define a homeomorphism $h$ of $\mathbb{R}^{d}$ onto itself by

$$
h(t)=\left(\varphi\left(t_{1}\right), \varphi\left(t_{2}\right), \ldots, \varphi\left(t_{d}\right)\right), \quad t=\left(t_{1}, t_{2}, \ldots, t_{d}\right) \in \mathbb{R}^{d} .
$$

Consider an arbitrary function $f \in K$ and set $g=f \circ h$. Let us verify that $g \in \bigcap_{1<p<\infty} M_{p}\left(\mathbb{R}^{d}\right)$. Given a rectangle $I \subset \mathbb{R}^{d}$ denote the center of $I$ by $c_{I}$. For $\nu=1,2, \ldots$, let $g_{\nu}$ be the function that takes the constant value $g\left(c_{I}\right)$ on each rectangle $I \in \alpha_{d}(\nu)$. Clearly,

$$
\left\|g-g_{\nu}\right\|_{L^{\infty}\left(\mathbb{R}^{d}\right)} \leq \sup _{I \in \alpha_{d}(\nu)} \operatorname{Osc}_{I} g .
$$

Note that if $I \in \alpha_{d}(\nu)$, then the image $h(I)$ of $I$ under $h$ is a certain rectangle whose edges are of length at most $\delta_{\nu}$, which implies that $\operatorname{diam} h(I) \leq \delta_{\nu} \sqrt{d}$. So,

$\operatorname{OSc}_{I} g=\operatorname{osc}_{h(I)} f \leq \omega\left(f, \delta_{\nu} \sqrt{d}\right) \leq \omega\left(\delta_{\nu} \sqrt{d}\right) \quad$ for all $I \in \alpha_{d}(\nu), \quad \nu=1,2, \ldots$ Therefore (see (11)),

$$
\left\|g-g_{\nu}\right\|_{L^{\infty}\left(\mathbb{R}^{d}\right)} \leq \omega\left(\delta_{\nu} \sqrt{d}\right)
$$


Hence, for $\nu \geq 2$, we obtain

$$
\left\|g_{\nu}-g_{\nu-1}\right\|_{L^{\infty}\left(\mathbb{R}^{d}\right)} \leq\left\|g_{\nu}-g\right\|_{L^{\infty}\left(\mathbb{R}^{d}\right)}+\left\|g-g_{\nu-1}\right\|_{L^{\infty}\left(\mathbb{R}^{d}\right)} \leq 2 \omega\left(\delta_{\nu-1} \sqrt{d}\right) .
$$

Let $1<p<\infty$. Since $g_{\nu} \in \mathcal{P}_{\nu}$, it follows that $g_{\nu} \in M_{p}\left(\mathbb{R}^{d}\right), \nu=1,2, \ldots$ Note that $g_{\nu}-g_{\nu-1} \in \mathcal{P}_{\nu}$ for $\nu \geq 2$; therefore (see (7) and (13)), we have

$$
\left\|g_{\nu}-g_{\nu-1}\right\|_{M_{p}\left(\mathbb{R}^{d}\right)} \leq c(p, \nu) 2 \omega\left(\delta_{\nu-1} \sqrt{d}\right)
$$

Thus,

$$
\left\|g_{n+m}-g_{n}\right\|_{M_{p}\left(\mathbb{R}^{d}\right)} \leq \sum_{\nu=n+1}^{n+m}\left\|g_{\nu}-g_{\nu-1}\right\|_{M_{p}\left(\mathbb{R}^{d}\right)} \leq \sum_{\nu=n+1}^{n+m} 2 c(p, \nu) \omega\left(\delta_{\nu-1} \sqrt{d}\right) .
$$

Taking (9) into account, we see that the sequence $g_{\nu}, \nu=1,2, \ldots$, converges in $M_{p}\left(\mathbb{R}^{d}\right)$ (recall that $M_{p}$ is a Banach space). At the same time from (12) it follows that this sequence converges to $g$ in $L^{\infty}\left(\mathbb{R}^{d}\right)$. It remains to recall that $\|\cdot\|_{L^{\infty}}=\|\cdot\|_{M_{2}} \leq\|\cdot\|_{M_{p}}$. This completes the proof of Theorem 1 .

Proof of Theorem 2. Let $K$ be a uniformly equicontinuous family of functions on $\mathbb{T}^{d}$. We identify each function on $\mathbb{T}^{d}$ with a $2 \pi$-periodic (in each variable) function on $\mathbb{R}^{d}$ in the standard manner. Thus, $K$ is a family of bounded functions uniformly equicontinuous on $\mathbb{R}^{d}$. Following the proof of Theorem 1 (now we do not need the Lemma), we obtain a selfhomeomorphism $\varphi$ of $\mathbb{R}$ such that defining self-homeomorphism $h$ of $\mathbb{R}^{d}$ by (10) we have $f \circ h \in \bigcap_{1<p<\infty} M_{p}\left(\mathbb{R}^{d}\right)$ for all $f \in K$. Consider the interval $J=\varphi^{-1}([0,2 \pi])$ which is the preimage of $[0,2 \pi]$ under $\varphi$. Let $l$ be an affine self-mapping of the real line for which $l([0,2 \pi])=J$. We set $\varphi_{1}=\varphi \circ l$ and

$$
h_{1}(x)=\left(\varphi_{1}\left(x_{1}\right), \varphi_{1}\left(x_{2}\right), \ldots, \varphi_{1}\left(x_{d}\right)\right), \quad x=\left(x_{1}, x_{2}, \ldots, x_{d}\right) \in \mathbb{R}^{d} .
$$

Using the assertion on superpositions of multipliers with affine mappings (see Section 3, C), we conclude that $f \circ h_{1} \in \bigcap_{1<p<\infty} M_{p}\left(\mathbb{R}^{d}\right)$ for all $f \in K$. Recall that the indicator function of any rectangle in $\mathbb{R}^{d}$ is a multiplier for all $p, 1<p<\infty$ (see Section 3, B); hence

$$
1_{[0,2 \pi]^{d}} \cdot\left(f \circ h_{1}\right) \in \bigcap_{1<p<\infty} M_{p}\left(\mathbb{R}^{d}\right)
$$


for all $f \in K$. Since the homeomorphism $\varphi_{1}$ maps the interval $[0,2 \pi]$ onto itself, we can regard it as a self-homeomorphism of the circle $\mathbb{T}$. So, we can regard $h_{1}$ as a self-homeomorphism of the torus $\mathbb{T}^{d}$. Denote this selfhomeomorphism of the torus by $h_{2}$. Clearly, $f \circ h_{2}$ is a $2 \pi$-periodic in each variable extension of the function $1_{[0,2 \pi]^{d}} \cdot\left(f \circ h_{1}\right)$. It remains to use the theorem on periodic extensions (see Section 3,D). This completes the proof of Theorem 2.

\section{Remarks and Open Problems}

1. As we stated in Introduction, the negative solution of Luzin's problem implies a similar result for functions on the real line, namely: there exists a bounded continuous real-valued function $f$ on $\mathbb{R}$ such that $f \circ h \notin B(\mathbb{R})$ for every self-homeomorphism $h$ of $\mathbb{R}$. One can easily verify this as follows. Let $f$ be a function on $\mathbb{T}$ which provides the negative solution of Luzin's problem. Regarding $f$ as a $2 \pi$-periodic function on $\mathbb{R}$, we can assume without loss of generality that $f(0)=f(2 \pi)=0$. Let $f_{0}$ be the function on $\mathbb{R}$ that coincides with $f$ on the interval $[0,2 \pi]$ and vanishes outside it. Suppose that $f_{0} \circ h \in B(\mathbb{R})$ for some self-homeomorphism $h$ of $\mathbb{R}$. Clearly, if a function belongs to $B(\mathbb{R})$, then so does every superposition of this function with an affine mapping $l: \mathbb{R} \rightarrow \mathbb{R}$; so, replacing $h$ by $h \circ l$ if necessary, we can assume that $h$ maps $[0,2 \pi]$ onto itself, and hence $f_{0} \circ h$ vanishes outside $[0,2 \pi]$. It follows that $2 \pi$-periodic extension of $f_{0} \circ h$ to $\mathbb{R}$ is in $A(\mathbb{T})$ (see Section 3, D), which is impossible, since this extension has the form $f \circ h_{1}$, where $h_{1}$ is a self-homeomorphism of the circle.

2. It is natural to consider an analogue of Luzin's problem in the multidimensional case. Is it true that, given a real-valued function $f \in C\left(\mathbb{T}^{d}\right), d \geq$ 2 , there exists a self-homeomorphism $h$ of $\mathbb{T}^{d}$ such that $f \circ h \in A\left(\mathbb{T}^{d}\right)$ ? Since the group of homeomorphisms of $\mathbb{T}^{d}$ with $d \geq 2$ is more massive than that of $\mathbb{T}$ the question may have a positive answer for $d \geq 2$ despite the fact that in the one-dimensional case it is answered in the negative.

3. As in the introduction, let $G$ be either $\mathbb{R}^{d}$ or $\mathbb{Z}^{d}$ and $\Gamma$ is $\mathbb{R}^{d}$ or $\mathbb{T}^{d}$, correspondingly. Recall that a function $m \in L^{\infty}(\Gamma)$ is called a weak type $(1,1)$ multiplier if the operator $Q$ defined by $\widehat{Q f}=m \widehat{f}, f \in L^{1} \cap L^{2}(G)$, is of weak type $(1,1)$, i.e., satisfies the condition

$$
\operatorname{mes}_{G}\{t \in G:|Q f(t)|>\lambda\} \leq c\|f\|_{L^{1}(G)} / \lambda, \quad \lambda>0,
$$


where $\operatorname{mes}_{G}$ is the Haar measure on $G$ (the Lebesgue measure of a set in the case of $G=\mathbb{R}^{d}$ and the number of elements in a set in the case of $\left.G=\mathbb{Z}^{d}\right)$. Let $M_{1}^{\text {weak }}(\Gamma)$ denote the class of all such multipliers. We have (using Marcinkiewicz' interpolation theorem)

$$
M_{1}(\Gamma) \subseteq M_{1}^{\text {weak }}(\Gamma) \subseteq \bigcap_{1<p<\infty} M_{p}(\Gamma)
$$

The authors do not know whether it is possible to improve Theorems 1 and 2 so as to attain the condition $f \circ h \in M_{1}^{\text {weak }}$ for all $f \in K$. The answer is unclear even for the families that consist of one function: Given a bounded real-valued function $f \in C\left(\mathbb{R}^{d}\right)$ or a real-valued function $f \in C\left(\mathbb{T}^{d}\right)$ is there a homeomorphic change of variable $h$ such that $f \circ h \in M_{1}^{\text {weak }}$ ? The negative answer to this question in the one-dimensional case would strengthen the result that solves Lusin's problem.

4. Let $d \geq 2$. Observe that the homeomorphism $h$ of $\mathbb{T}^{d}$ in Theorem 2 can be chosen in the form

$$
h:\left(t_{1}, t_{2}, \ldots, t_{d}\right) \rightarrow\left(\varphi\left(t_{1}\right), \varphi\left(t_{2}\right), \ldots, \varphi\left(t_{d}\right)\right),
$$

where $\varphi$ is a self-homeomorphism of $\mathbb{T}$. As concerns Theorem 1 , it is clear from its proof that if we impose stronger assumption on $f$ or on $K$, namely if we assume that $f$ is bounded and uniformly continuous or, respectively, that $K$ is a family of bounded functions which is uniformly equicontinuous on the whole $\mathbb{R}^{d}, d \geq 2$, then the corresponding homeomorphism $h$ of $\mathbb{R}^{d}$ can be chosen in the form (15) with $\varphi$ being a self-homeomorphism of $\mathbb{R}$. We do not know if the same is true without the above stronger assumptions. Moreover, it is unclear if every bounded real-valued function $f \in C\left(\mathbb{R}^{d}\right)$ can be transformed into a multiplier by a homeomorphism of the form $h:\left(t_{1}, t_{2}, \ldots, t_{d}\right) \rightarrow\left(\varphi_{1}\left(t_{1}\right), \varphi_{2}\left(t_{2}\right), \ldots, \varphi_{d}\left(t_{d}\right)\right)$, where $\varphi_{j}$ 's are selfhomeomorphisms of $\mathbb{R}$ allowed to be different. It seems likely that the answer is negative.

5. The well-known Beurling-Helson theorem [2] (see also [8, Ch. VI], [9]) states that if $\varphi$ is a continuous self-mapping of the circle $\mathbb{T}$ satisfying the condition $\left\|e^{i n \varphi}\right\|_{A(\mathbb{T})}=O(1), n \in \mathbb{Z}$, then $\varphi$ is linear (affine), i.e., $\varphi(t)=\nu t+\varphi(0)$ where $\nu \in \mathbb{Z}$. The character of growth of the norms $\left\|e^{i n \varphi}\right\|_{A(\mathbb{T})}$ for nontrivial $\varphi$ 's is unclear in many respects. Kahane conjectured that the Beurling-Helson theorem can be considerably improved; namely, he conjectured in [7] (see also [8, Ch. VI], [9]) that the conclusion of the 
Beurling-Helson theorem remains valid even if the norms $\left\|e^{i n \varphi}\right\|_{A(\mathbb{T})}$ grow to infinity but the growth is not very fast. He also conjectured ([7], [8]) that the condition $\left\|e^{i n \varphi}\right\|_{A(\mathbb{T})}=o(\log |n|)$ already implies linearity of $\varphi$. The first result in this direction was obtained in [17]; further strengthening is obtained in [12], however the $o(\log |n|)$-conjecture remains unproved.

Certain analogs of the Beurling-Helson theorem for the algebras $M_{p}$ were obtained in [20] and [21]. Note that the case $1<p<\infty$ differs from that of $p=1$, which corresponds to the Wiener algebra; for example (see [5, Ch. I, Sec. 1.3]), if $\varphi: \mathbb{T} \rightarrow \mathbb{T}$ is piecewise linear, then for all $p, 1<p<\infty$,

$$
\left\|e^{i n \varphi}\right\|_{M_{p}(\mathbb{T})}=O(1), \quad n \in \mathbb{Z} .
$$

To some extent the converse is also true [21, Theorem $\left.2^{\prime}\right]$ : if (16) holds for some $p \neq 2$, then there exists a closed set $E(\varphi) \subset \mathbb{T}$ of Lebesgue measure zero such that $\varphi$ is linear on the intervals complementary to $E(\varphi)$, and the set of distinct slopes of $\varphi$ is finite.

It is natural to ask how slow the norms $\left\|e^{i n \varphi}\right\|_{M_{p}}$ can grow in the case when $\varphi$ is nowhere (i.e., on no interval) linear. The answer to this question can easily be extracted from results of this paper. For $1<p<\infty$ the growth can be arbitrarily slow, namely: given an arbitrary positive sequence $\gamma(n), n=0,1,2, \ldots$, with $\gamma(n) \rightarrow+\infty$, there exists a nowhere linear selfhomeomorphism $h$ of $\mathbb{T}$ such that

$$
\left\|e^{i n h}\right\|_{M_{p}(\mathbb{T})}=O(\gamma(|n|)), \quad n \in \mathbb{Z},
$$

for all $p, 1<p<\infty$.

To prove this assertion, we firstly observe that Theorems 1 and 2 can be supplemented by the estimate $\|f \circ h\|_{M_{p}} \leq c(p, 1)\|f\|_{L^{\infty}}+c_{p}(K), f \in K$. In particular, if $K$ is a compact set in the space $C\left(\mathbb{T}^{d}\right)$, then there exists a homeomorphism $h: \mathbb{T}^{d} \rightarrow \mathbb{T}^{d}$ such that

$$
\|f \circ h\|_{M_{p}\left(\mathbb{T}^{d}\right)} \leq c_{K}(p), \quad 1<p<\infty, \quad f \in K .
$$

Indeed (see the end of the proof of Theorem 1), since $g_{\nu} \stackrel{M_{p}}{\rightarrow} g$, it follows from (14) that $\left\|g-g_{1}\right\|_{M_{p}} \leq 2 c_{p}(K)$, where $c_{p}(K)$ is the sum of the series in (9). At the same time $\left\|g_{1}\right\|_{M_{p}} \leq c(p, 1)\left\|g_{1}\right\|_{L^{\infty}} \leq c(p, 1)\|f\|_{L^{\infty}}$.

Secondly we observe that due to sufficient flexibility in the construction of the net $\beta$ in the proof of Theorem 1, the homeomorphism $h$ in the statement of Theorems 1 and 2 can be made nowhere linear. 
It remains to apply the above two observations to the family

$$
K=\left\{\frac{e^{i n t}}{\gamma(|n|)}, \quad n \in \mathbb{Z}\right\}
$$

(see (17)).

\section{References}

1. N. K. Bary, A treatise on trigonometric series, Vols. I, II, Pergamon Press, Oxford 1964.

2. A. Beurling, H. Helson, "Fourier-Stieltjes transforms with bounded powers", Math. Scand., 1 (1953), 120-126.

3. R. E. Edwards and G. I. Gaudry, Littlewood-Paley and multiplier theory. Springer-Verlag, Berlin-Heidelberg, 1977.

4. G. Goffman, T. Nishiura, D. Waterman, Homeomorphisms in Analysis, Mathematical Surveys and Monographs v. 54, Amer. Math. Soc., 1997.

5. L. Hörmander, "Estimates for translation invariant operators in $L^{p}$ spaces", Acta Math., 104 (1960), 93-140.

6. M. Jodeit, "Restrictions and extensions of Fourier multipliers", Studia Math., 34 (1970), 215-226.

7. J.-P. Kahane, "Transformées de Fourier des fonctions sommables", Proceedings of the Int. Congr. Math., 15-22 Aug., 1962, Stockholm, Sweden, Inst. Mittag-Leffler, Djursholm, Sweden, 1963, pp. 114-131.

8. J.-P. Kahane, Série de Fourier absolument convergentes, Springer-Verlag, Berlin-Heidelberg-New York, 1970.

9. J.-P. Kahane, "Quatre leçons sur les homéomorphismes du circle et les séries de Fourier", in: Topics in Modern Harmonic Analysis, Vol. II, Ist. Naz. Alta Mat. Francesco Severi, Roma, 1983, 955-990.

10. J.-P. Kahane, Y. Katznelson, "Homéomorphismes de cercle et séries de Fourier absolument convergent", Compt. Rend., Paris, 292:4 (1981), Série I, 271-273. 
11. J.-P. Kahane, Y. Katznelson, "Séries de Fourier des fonctions bornée", Studies in Pure Math., in Memory of Paul Turán, Budapest, 1983, pp. 395-410. (Preprint, Orsay, 1978.)

12. S. V. Konyagin and I. D. Shkredov, "A quantitative version of the Beurling-Helson theorem," Funct. Anal. Appl. 49:2 (2015), 110-121.

13. G. Kozma, "Random homeomorphisms and Fourier expansions the pointwise behaviour", Israel Journal of Mathematics, 139 (2004), 189-213.

14. G. Kozma and A. M. Olevskii, "Random homeomorphisms and Fourier expansions", Geometric and Fnnctional Analysis, 8 (1998), 1016-1042.

15. V. V. Lebedev, "Change of variable and the rapidity of decrease of Fourier coefficients", Matematicheskic Sbornik, 181:8 (1990), 1099-1113 (in Russian). English transl.: Mathematics of the USSR-Sbornik, 70:2 (1991), 541-555. English transl. corrected by the author is available at: https://arxiv.org/abs/1508.06673

16. V. V. Lebedev, "Torus homeomorphisms, Fourier coefficients, and integral smoothness", Russian Mathematics (Iz. VUZ), 36:12 (1992), 36-41.

17. V. V. Lebedev, "Absolutely convergent Fourier series. An improvement of the Beurling-Helson theorem," Funct. Anal. Appl. 46:2 (2012), 121-132.

18. V. Lebedev, "The Bohr-Pál theorem and the Sobolev space $W_{2}^{1 / 2}$ ", Studia Mathematica, 231:1 (2015), 73-81.

19. V. V. Lebedev, "A short and simple proof of the Jurkat-Waterman theorem on conjugate functions", Functional Analysis and Its Applications, 51:2 (2017), 148-151.

20. V. Lebedev and A. Olevskiǐ, " $C^{1}$ changes of variable: Beurling-Helson type theorem and Hörmander conjecture on Fourier multipliers", Geometric and Functional Analysis (GAFA) 4 (1994), 213-235. 
21. V. V. Lebedev, A. M. Olevskiǐ, " $L^{p}$-Fourier multipliers with bounded powers", Izvestya: Mathematics, 70:3 (2006), 549-585.

22. A. M. Olevskiǐ, "Change of variable and absolute convergence of Fourier series", Soviet Math. Dokl., 23 (1981), 76-79.

23. A. M. Olevskiǐ, "Modifications of functions and Fourier series", Russian Math. Surveys, 40 (1985), 181-224.

24. G. T. Oniani, "Topological characterization of a set of continuous functions whose conjugate functions are continuous and have bounded variation", Trudy Tbiliss. Mat. Inst. Razmadze Akad. Nauk Gruzin. SSR, 86 (1987), 110-113 (in Russian). English transl.: Integral Operators and Boundary Properties of Functions. Fourier Series, 155-161, Nova Science, New York, 1992.

25. A. A. Saakjan, "Integral moduli of smoothness and the Fourier coefficients of the composition of functions", Matematicheskir Sbornik, 110(152):4(12) (1979), 597-608 (in Russian). Engl. transl.: Mathematics of the USSR-Sbornik, 38 (1981), 549-561.

26. P. Sjögren and P. Sjölin, "Littlewood-Paley decompositions and Fourier multipliers with singularities on certain sets.", Ann. Inst. Fourier, Grenoble, 31 (1981), 157-175.

Vladimir Lebedev

School of Applied Mathematics

National Research University Higher School of Economics

34 Tallinskaya St.

Moscow, 123458 Russia

E-mail address: lebedevhome@gmail.com

Alexander Olevskii

School of Mathematical Sciences

Sackler Faculty of Exact Sciences

Tel Aviv University

Tel Aviv, 69978 Israel

E-mail address: olevskii@yahoo.com 